\section{Diagnóstico precoz de cáncer gástrico. Propuesta de detección y seguimiento de lesiones premalignas gástricas: protocolo ACHED}

\author{
ANTONIO ROLLÁN ${ }^{1}$, PABLO CORTÉS ${ }^{1}$, ALFONSO CALVO $^{2}$, \\ RAÚLARAYA ${ }^{3}$, MARÍA ESTER BUFADEL ${ }^{4}$, ROBINSON GONZÁLEZ $^{5}$, \\ CAROLINA HEREDIA ${ }^{6}$, PABLO MUNOOZ ${ }^{7}$, FREDDY SQUELLA $^{8}$, \\ ROBERTO NAZAL ${ }^{9}$, MARÍA DE LOS ÁNGELES GATICA ${ }^{1}$, \\ JAQUELINA GOBELET ${ }^{1}$, RENÉ ESTAY $^{10}$, RAÚL PISANO $^{11}$, \\ LUIS CONTRERAS $^{12}$, INGRID OSORIO ${ }^{13, \mathrm{a}}$, RICARDO ESTELA $^{14}$, \\ FERNANDO FLUXÁ ${ }^{15}$, ADOLFO PARRA-BLANCO
}

\section{Recommendations of the Chilean association for digestive endoscopy for the management of gastric pre-malignant lesions}

An expert panel analyzed the available evidence and reached a consensus to release 24 recommendations for primary and secondary prevention of gastric cancer (CG) in symptomatic patients, with indication for upper GI endoscopy. The main recommendations include (1) Search for and eradicate H. pylori infection in all cases. (2) Systematic gastric biopsies (Sydney protocol) in all patients over 40 years of age or first grade relatives of patient with CG, to detect gastric atrophy, intestinal metaplasia or dysplasia. (3) Incorporate the OLGA system (Operative Link on Gastritis Assessment) to the pathological report, to categorize the individual risk of CG. (4) Schedule endoscopic follow-up according to the estimated risk of CG, namely annual for OLGA III-IV, every 3 years for OLGA I- II or persistent $H$. pylori infection, every 5 years for $C G$ relatives without other risk factors and no follow-up for OLGA 0, H. pylori (-). (4) Establish basic human and material resources for endoscopic follow-up programs, including some essential administrative processes, and (5) Suggest the early CG/total CG diagnosis ratio of each institution and the proportion of systematic recording of endoscopic images, as quality indicators. These measures are applicable using currently available resources, they can complement any future screening programs for asymptomatic population and may contribute to improve the prognosis of CG in high-risk populations.

(Rev Med Chile 2014; 142: 1181-1192)

Key words: Gastric neoplasms; Health planning guidelines; Mass screening.
'Unidad de Gastroenterología, Clínica Alemana de Santiago. Facultad de Medicina Clínica Alemana-Universidad del Desarrollo, Santiago, Chile.

${ }^{2}$ Unidad de Endoscopia CRS San Rafael y Endoscopia Terapéutica Hospital Dr. Sótero del Río, Santiago, Chile. ${ }^{3}$ Unidad de Gastroenterología y Servicio de Endoscopia. Hospital Militar de Santiago, Chile.

${ }^{4}$ Sección de Gastroenterología, Hospital Clínico Universidad de Chile y Clínica Avansalud, Integramédica, Santiago, Chile.

${ }^{5}$ Departamento de Gastroenterología, Facultad de Medicina, Pontificia Universidad Católica de Chile, Santiago, Chile.

${ }^{6}$ Servicio de Endoscopia, Hospital Militar de Santiago, Facultad de Medicina, Universidad de los Andes, Santiago, Chile. ${ }^{7}$ Sección de Gastroenterología. Hospital Clínico Universidad de Chile, Santiago, Chile.

${ }^{8}$ Servicio de Gastroenterología Clínica Indisa y Universidad Andrés Bello, Santiago, Chile.

${ }^{9}$ Hospital San José e Integramédica, Santiago, Chile.

${ }^{10}$ Clínica Santa María y Hospital del Salvador, Santiago, Chile.

${ }^{11}$ Unidad de Anatomía Patológica, Hospital San Juan de Dios, Santiago, Chile.

${ }^{12}$ Laboratorio de Anatomía Patológica Cy S, Santiago, Chile. ${ }^{13}$ Hospital El Pino, Santiago, Chile. ${ }^{14}$ Instituto Chileno-Japonés de Enfermedades Digestivas. Hospital Clínico San Borja-Arriarán. Departamento de Medicina Centro. Facultad de Medicina. Universidad de Chile, Santiago, Chile. 15 Departamento de Gastroenterología. Clinica Las Condes, Santiago, Chile. aEnfermera Universitaria.

Recibido el 23 de enero de 2014, aceptado el 11 de agosto de 2014

Correspondencia a: Dr. Antonio Rollán Unidad de Gastroenterología. Clínica Alemana de Santiago Av. Vitacura 5951. Tercer piso. Santiago. arollan@alemana.cl 
A pesar de una leve disminución de frecuencia ${ }^{1}$, el cáncer gástrico (CG) continúa siendo la primera causa de muerte por cáncer en nuestro país. El 90\% de los casos diagnosticados son avanzados, con una sobrevida global de $10 \%$ a 5 años ${ }^{2,3}$.

El protocolo AUGE (Acceso Universal con Garantías Explícitas) del Ministerio de Salud de Chile para CG (http://www.minsal.cl/portal/url/ item/722233c6b943cd08e04001011f011d5e.pdf) no considera la detección ni el seguimiento de pacientes con lesiones premalignas gástricas. Sólo una estrategia específica de diagnóstico precoz puede modificar el pronóstico de esta enfermedad.

La hipótesis más aceptada propone la gastritis crónica por $H$. pylori como factor etiológico inicial del $C^{4,5}$. La lenta progresión a atrofia gástrica (AG), metaplasia intestinal (MI), displasia y adenocarcinoma invasor (de tipo intestinal) es modulada a lo largo de muchos años por factores genéticos, dietéticos y ambientales, ofreciendo una amplia oportunidad de intervención.

Programas parciales de tamizaje poblacional de CG sólo existen en algunos países asiáticos ${ }^{6-8}$. La conducta más recomendada en el mundo es el tamizaje oportuno. Experiencias previas en Chile han mostrado tasas de detección de CG de 1,2\% y $1,3 \%$ en pacientes sintomáticos ${ }^{9,10}$, con hasta $20 \%$ de CG incipiente ${ }^{10}$. Un programa de detección y seguimiento de lesiones premalignas podría mejorar el pronóstico del CG en Chile.

\section{Objetivos}

Establecer recomendaciones de detección y seguimiento de condiciones premalignas gástricas, para mejorar la detección de CG incipiente y el pronóstico del CG en nuestro país.

\section{Métodos}

La Asociación Chilena de Endoscopia Digestiva (ACHED) convocó un grupo de gastroenterólogos, cirujanos digestivos, patólogos y enfermeras interesados en CG. Se conformaron grupos de trabajo, que realizaron una revisión sistemática de la literatura para responder las siguientes preguntas: 1. ¿Cuáles son y cómo detectar condiciones/ lesiones gástricas premalignas durante una Endoscopia Digestiva Alta (EDA)?

2. ¿Cómo categorizar el riesgo futuro de CG en los pacientes sometidos a EDA?
3. ¿Quiénes deben incluirse en un programa de seguimiento endoscópico?

4. ¿Qué características debe tener una EDA de seguimiento para detectar CG incipiente?

5. ¿Qué características debe tener un programa de seguimiento endoscópico para pacientes con alto riesgo de $\mathrm{CG}$ ?

Cada grupo formuló recomendaciones, con nivel de evidencia y grado de recomendación establecidos de acuerdo a la U.S. Preventive Services Task Force (Tablas 1 y 2) ${ }^{11,12}$.

El consenso se obtuvo utilizando el método Delphi ${ }^{13}$. Las recomendaciones fueron evaluadas por los miembros del panel mediante una escala Likert: 1) Completamente en desacuerdo; 2) En desacuerdo; 3) Dudoso o con reparos; 4) De acuerdo y 5) Completamente de acuerdo. En caso de desacuerdo se reformuló y votó nuevamente la recomendación. Se presentan y fundamentan las que obtuvieron un acuerdo promedio final $\geq 4$.

\section{Resultados}

\section{Detección y manejo de lesiones premalignas gástricas}

\section{Recomendaciones}

1. Se sugiere detectar y erradicar H. pylori en todos los pacientes sometidos a EDA. Esto ofrece la posibilidad de mejoría sintomática, reduce la progresión a AG/MI y el riesgo futuro de CG (Evidencia I; Recomendación A).

2. Se sugiere buscar AG, MI o displasia en todo paciente sintomático mayor de 40 años o de cualquier edad con antecedentes familiares de CG. (Evidencia II, Recomendación B).

3. El diagnóstico de lesiones premalignas requiere histología. Se recomienda el protocolo de Sydney. Las biopsias deben enviarse separadas en dos frascos (antro-ángulo y cuerpo) (Evidencia II, Recomendación B).

4. La identificación de lesiones focales puede realizarse con endoscopia de alta resolucion y luz blanca. Su caracterización mejora con magnificación óptica y cromoendoscopia (con tinciones o digital) (Evidencia II; Recomendación B).

Las condiciones premalignas gástricas incluyen la infección por H. pylori, la AG, la MI y la displa- 
Protocolo ACHED para manejo de lesiones premalignas gástricas - A. Rollán et al

Tabla 1. Niveles de Evidencia

\begin{tabular}{|cl|}
\hline $\begin{array}{c}\text { Nivel de } \\
\text { evidencia }\end{array}$ & Descripción \\
Tipo I & $\begin{array}{l}\text { Evidencia obtenida de al menos un estudio controlado* randomizado bien diseñado o una revisión siste- } \\
\text { mática de estudios clínicos randomizados }\end{array}$ \\
Tipo II & $\begin{array}{l}\text { II-1 Evidencia obtenida de estudios controladosprospectivos sin randomización } \\
\text { II-2 Evidencia obtenida de estudios observacionales** de tipo cohorte o casos y controles, idealmente de } \\
\text { varios centros } \\
\text { II-3 Evidencia obtenida de series de casos }\end{array}$ \\
& $\begin{array}{l}\text { Opinión de autoridades en el tema basados en la experiencia, comités de expertos, reporte de casos, estu- } \\
\text { dios fisiopatológicos o de ciencia básica }\end{array}$ \\
\hline
\end{tabular}

*Aquel en que la intervención es manejada por el investigador. **Aquel en que la intervención no es controlada por el investigador.

Tabla 2. Niveles de Recomendación

\begin{tabular}{|cl|}
\hline Recomendación & Lenguaje \\
A & $\begin{array}{l}\text { El consenso recomienda fuertemente la intervención o servicio indicado. Esta recomendación se sus- } \\
\text { tenta en evidencia de alta calidad, con beneficio categórico que supera los riesgos }\end{array}$ \\
B & $\begin{array}{l}\text { El consenso recomienda el uso clínico rutinario de la intervención o servicio indicado. La recomenda- } \\
\text { ción se sustenta en evidencia de calidad moderada, con beneficio que supera los riesgos }\end{array}$ \\
C & $\begin{array}{l}\text { El consenso no recomienda ni a favor ni en contra de la intervención o servicio. No se realiza una } \\
\text { recomendación categórica dado que la evidencia, de calidad al menos moderada, no muestra una } \\
\text { relación riesgo/beneficio satisfactoria. Se debe decidir caso a caso }\end{array}$ \\
& $\begin{array}{l}\text { El consenso recomienda en contra de la intervención o servicio. La recomendación se sustenta en } \\
\text { evidencia de calidad al menos moderada que no muestra beneficio o que el riesgo o daño supera los } \\
\text { beneficios de la intervención } \\
\text { E consenso concluye que la evidencia es insuficiente, ya sea por estudios de baja calidad, resultados } \\
\text { heterogéneos, o el balance riesgo/beneficio no puede ser determinado }\end{array}$ \\
\hline
\end{tabular}

sia. El rol etiológico de $H$. pylori es indiscutido ${ }^{14}$. Con la excepción del CG hereditario difuso, el CG es prácticamente inexistente sin infección por H. pylori (pasada o actual) ${ }^{15,16}$. Su erradicación disminuye la incidencia de CG (prevención primaria ${ }^{17}$ y la de lesiones metacrónicas en pacientes tratados por CG (prevención secundaria) ${ }^{18}$. En Chile, la frecuencia de $H$. pylori alcanza $73 \pm 3 \%$ en población general ${ }^{19}$ y $78 \%$ en pacientes sintomáticos de la zona sur de Santiago ${ }^{20}$. Se sugiere realizar test de ureasa rápido y/o histología en todo paciente sometido a EDA. La erradicación ofrece la posibilidad de mejoría sintomática en pacientes dispépticos y disminuye el riesgo futuro de $\mathrm{CG}^{21,22}$.

Si se justifica detectar H. pylori, con mayor razón debe detectarse AG, MI o displasia, asociadas a un riesgo de CG mucho mayor ${ }^{16}$. La frecuencia de AG es muy baja antes de los 40 años $(<5 \%)^{23}$. En ausencia de lesiones focales o antecedentes familiares, la búsqueda de lesiones premalignas podría focalizarse en los mayores de 40 años. Un reciente guía europea, sin embargo, propone hacerlo en todos los casos, sin tomar en consideración la edad ${ }^{24}$.

Los familiares de primer grado de pacientes con CG tienen 2 a 10 veces más riesgo de $\mathrm{CG}^{25,26}$ y una mayor frecuencia y precocidad de $\mathrm{AG}^{27,28}$.

La endoscopia tiene mal rendimiento diagnóstico en occidente ${ }^{29,30}$, por lo que el diagnóstico de AG/ MI requiere biopsias sistemáticas del cuerpo $y_{\text {antro }}{ }^{24,31,32}$. El protocolo de Sydney modificado ${ }^{33}$ incluye 5 biopsias ( 2 en antro y cuerpo y una en el ángulo) y es el más aceptado. Un mayor número de biopsias pudiera aumentar la sensibilidad ${ }^{34}$. 
El objetivo principal de una EDA es explicar los síntomas y detectar CG incipiente. Las formas planas o deprimidas son difíciles de reconocer. Un endoscopista motivado y entrenado, con endoscopio de alta resolución, con luz blanca, es suficiente para detectar lesiones, aunque la cromoscopia, con tinciones o digital, puede mejorar su caracterización, dirigir las biopsias y precisar los límites de una eventual resección ${ }^{35}$.

\section{Categorización del riesgo de CG}

\section{Recomendaciones}

7. El riesgo de CG depende de la presencia de $H$. pylori y la presencia, intensidad y extensión de la AG, MI y displasia. Esto permite estimar la incidencia de CG y la necesidad de seguimiento endoscópico (Evidencia II, Recomendación B).

8. Se sugiere etapificar el riesgo de CG de acuerdo al sistema OLGA (Operative Link of Gastritis Asessment) para AG y/o MI (OLGIM), que debiera incluirse en el reporte histológico (Evidencia II, Recomendación B).

9. Se sugiere la clasificación de la OMS para displasia: 1) Negativo para displasia; 2) Indefinido para displasia; 3) Displasia de Bajo Grado (DBG); 4) Displasia de Alto grado (DAG); 5) Cáncer (Evidencia II, Recomendación B).

El CG puede aparecer cuando ya ha desaparecido la infección por $H$. pylori ${ }^{36}$. La gastritis de predominio corporal aumenta el riesgo y es más frecuente en poblaciones con alta frecuencia de
$\mathrm{CG}^{37}$, al igual que la presencia de AG/MI y displasia. En Japón, se ha descrito una gradiente de riesgo muy marcada: $0 \%$ anual en sujetos $H$. pylori (-), AG (-), 0,1\% anual en $H$. pylori (+), AG (-); $0,25 \%$ anual en $H$. pylori $(+)$; AG $(+)$ y $1 \%$ anual en $H$. pylori (-), AG $(+)^{16,38}$. En Holanda, un estudio de cohorte mostró una incidencia anual de $0,1 \%$ para $A G, 0,25 \%$ para $\mathrm{MI}, 0.6 \%$ para displasia leve a moderada, y $6 \%$ para displasia intensa ${ }^{39}$. $\mathrm{La}$ progresión anual de displasia a CG varía entre $0 \%$ y $73 \%$ en un año ${ }^{40}$, en parte debido a la variabilidad diagnóstica ${ }^{41}$. Existen diferencias sistemáticas en la valoración de displasia y CG entre patólogos orientales y occidentales ${ }^{42}$, que han motivado sucesivas propuestas de clasificación ${ }^{43}$. La recomendada por la $\mathrm{OMS}^{44}$ se resume en la Tabla 3.

El protocolo de Sydney no determina el riesgo individual de CG. Meining propuso un "Indice de riesgo de CG" basado en 3 criterios histológicos: inflamación de predominio corporal y MI presente en cuerpo y antro, (valores posibles de 0 a $3)$. En un estudio caso-control el score 3 tuvo un valor predictivo positivo de $94 \%$ para $\mathrm{CG}^{45}$. Un grupo de expertos propuso el "Sistema OLGA" (Operative Link of Gastritis Assessment) ${ }^{46}$, que considera 5 etapas con riesgo creciente (Tabla 4). Por la variabilidad en el diagnóstico de $\mathrm{AG}^{47}$, se ha sugerido reemplazar la AG por MI (Sistema OLGIM $)^{48}$, aunque esto pudiera disminuir levemente la sensibilidad ${ }^{49}$. Aún no existe validación directa de su capacidad predictiva ${ }^{50-54}$. Un estudio chino sugiere que la gastritis de predominio corporal y OLGIM II-IV se asocian significativamente al riesgo de $\mathrm{CG}^{55}$.

Tabla 3. Clasificación de Vienna (revisada) de las neoplasias epiteliales gastrointestinales

\begin{tabular}{|c|c|c|}
\hline Categoría & Diagnóstico & Manejo clínico \\
\hline 1 & Negativo para displasia & Seguimiento opcional \\
\hline 2 & Indefinida para displasia & Seguimiento \\
\hline 3 & $\begin{array}{l}\text { Neoplasia mucosa de bajo grado (BG) } \\
\text { Adenoma BG (Lesión focal) } \\
\text { Displasia BG (Sin lesión focal) }\end{array}$ & $\begin{array}{l}\text { Resección endoscópica } \\
\text { Seguimiento }\end{array}$ \\
\hline 4 & $\begin{array}{l}\text { Neoplasia mucosa de alto grado (AG) } \\
\text { 0.1. Adenoma/displasia } A G \\
\text { 0.2. Carcinoma no invasivo (Carcinoma in situ) } \\
\text { 0.3. Sospecha carcinoma invasivo } \\
\text { 0.4. Carcinoma intramucoso }\end{array}$ & Resección endoscópica o quirúrgica \\
\hline 5 & Carcinoma con invasión submucosa & Resección quirúrgica \\
\hline
\end{tabular}




\section{Programas de seguimiento endoscópico}

\section{Recomendaciones:}

10. La AG, la MI y la displasia constituyen condiciones/lesiones de alto riesgo para CG. Un seguimiento endoscópico programado aumenta la probabilidad de diagnosticar CG incipiente. (Evidencia II, Recomendación B). El intervalo de seguimiento debe ajustarse al riesgo estimado de CG (Evidencia III, Recomendación C).

11. Los pacientes con displasia de alto grado (DAG), sin lesiones focales, deben realizarse mapeo histológico extenso y seguimiento endoscópico cada 6 meses. Cualquier lesión focal debe ser resecada en bloque (mucosectomía o disección endoscópica submucosa) (Evidencia II, Recomendación B).

12. Los pacientes con displasia de bajo grado (DBG) sin lesión focal y aquellos con AG/MI extensa o intensa (OLGA III/IV) deben realizarse una EDA de seguimiento anual. Cualquier lesión focal debe ser resecada endoscópicamente (Evidencia III, Recomendación C).

13. Los pacientes con AG/MI leve a moderada (OLGA I/II) y aquellos con infección por $H$. pylori resistente pueden realizarse EDA de seguimiento cada 3 años (Evidencia III, Recomendación C).

15. Los familiares de primer grado de pacientes con CG, aun sin demostración de AG/MI o infección por $H$. pylori, pueden realizarse EDA de seguimiento cada 5 años (Evidencia III, recomendación $\mathrm{C}$ ).

16. Se sugiere retirar del programa de tamizaje endoscópico a los pacientes cuya expectativa de vida, por edad o comorbilidad asociada, se estime menor a 10 años (Evidencia II, Recomendación B).

La erradicación de H. pylori resuelve la gastritis $^{56}$. En ausencia de AG, el riesgo posterior de CG es tan bajo que no justifica seguimiento ${ }^{14}$, aunque la tasa de reinfección no ha sido bien estudiada en Chile. Un estudio mostró una tasa de reinfección de $13 \pm 4 \%$ a 3 años ${ }^{56}$.

El efecto de la erradicación de $H$. pylori sobre la AG y la MI es incierto ${ }^{57}$. La evidencia sugiere una lenta regresión de la AG, que requiere hasta 6 años, mientras la regresión de la MI parece menos probable ${ }^{58}$. Ha sido difícil demostrar una disminución en la incidencia de $\mathrm{CG}^{59}$, pero el meta-analisis más reciente, que incluyó mayoritariamente pacientes con AG, concluyó que la erradicación disminuye la incidencia de $\mathrm{CG}^{60}$. Probablemente existe un "punto de no retorno", de modo que, aún después de una erradicación exitosa, el seguimiento endoscópico es necesario ${ }^{61}$.

No hay estudios controlados que demuestren el beneficio de un programa de seguimiento endoscópico. En un estudio inglés, el seguimiento anual de sujetos con lesiones gástricas premalignas detectó más casos de CG (8,4\% vs 1,3\%), en etapas más precoces (Estadios I and II $67 \%$ vs 23\%; $\mathrm{p}<0,05)$ y con mejor sobrevida a largo plazo $(50 \%$ vs $10 \% ; p=0,006)$, en comparación con pacientes estudiados por indicación clínica ${ }^{62}$.

La frecuencia óptima de control no ha sido determinada $^{63}$. En Japón se ha propuesto EDA anual en pacientes $\mathrm{HP}(-) / \mathrm{AG}(+)$, cada 2 años en pacientes $\mathrm{Hp}(+) / \mathrm{AG}(+)$ y cada 3 años en $\mathrm{Hp}(+) /$ AG(-). Graham propone EDA anual para OLGA IV, cada dos años para OLGA III, cada 5 años para

Tabla 4. Etapificación del riesgo de cancer gástrico según histología: Sistema OLGA (Operative Link for Gastritis Asessment) (modificado de ref. 46)

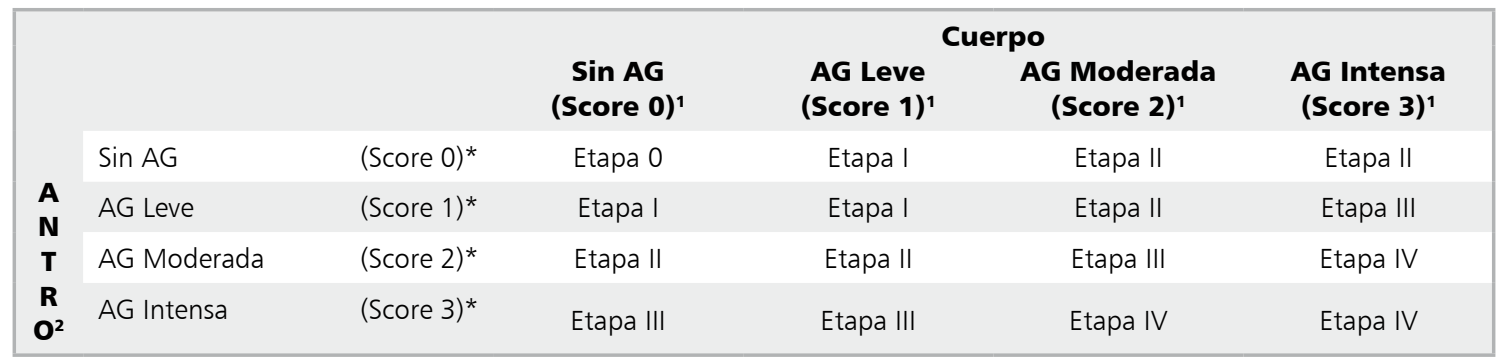

'Mediante Escala Visual Análoga, de acuerdo al Protocolo de Sydney. ${ }^{2}$ Incluye ángulo. 
OLGA II y no seguimiento para OLGA $0-\mathrm{I}^{14}$. Una guía europea reciente sugiere EDA cada dos años en pacientes con AG extensa y cada seis meses en pacientes con displasia ${ }^{24}$. En Corea, la mayor parte de los gastroenterólogos sugieren EDA anual en presencia de $A^{6}{ }^{64}$. Una revisión reciente sugiere EDA anual en pacientes con MI extensa, MI incompleta, tabaquismo o familiares con CG y cada 2 años para aquellos con MI sin estos factores ${ }^{65}$. Estudios de costo-efectividad norteamericanos (población con bajo riesgo de CG) justifican EDA cada 1-5 años en pacientes con displasia o luego de resección endoscópica ${ }^{66}$ y EDA anual en sujetos entre 60 y 70 años con MI extensa ${ }^{67}$.

La DAG implica alto riesgo de CG sincrónico. Toda lesión focal (adenoma) debe resecarse ${ }^{24,68}$. Cuando la displasia se detecta en biopsias sistemáticas, se recomienda mapeo histológico extenso o mucosectomía de la zona ${ }^{24}$ y requieren un seguimiento muy cercano ${ }^{39}$. Nuestra propuesta se resume en la Tabla 5.

En programas de tamizaje para otras neoplasias se ha sugerido que el beneficio no justifica los costos y riesgo asociados cuando la expectativa de vida estimada es menor a 10 años ${ }^{69-71}$, especialmente si se utilizan procedimientos invasivos. Este tema no ha sido abordado específicamente para CG.

\section{Requisitos de EDA de seguimiento}

\section{Recomendaciones:}

17. Una capacitación específica mejora el diagnóstico de CG incipiente. Los aspectos claves incluyen el examen completo del estómago y la búsqueda dirigida de lesiones planas. (Evidencia II, Recomendación B).

18. Debe utilizarse el mejor endoscopio disponible. (Evidencia III, Recomendación C). La técnica básica es endoscopia de alta resolución con luz blanca y cromoscopia con índigo carmín. Cuando sea posible, se recomienda utilizar magnificación óptica y cromoendoscopia digital (Evidencia II, Recomendación B).

19. El uso previo de simeticona y/o acetil-cisteína mejora la visualización de la mucosa gástrica y facilita la exploración (Evidencia I, Recomendación B).

20. Se recomiendan biopsias sistemáticas (Sydney) en cada EDA de tamizaje, para actualizar el riesgo futuro de CG. Las biopsias deben ser remitidas inequívocamente identificadas (Evidencia II, Recomendación B).

21. Las imágenes a registrar incluyen toda lesión focal y registro fotográfico sistemático que confirme la exploración gástrica completa. Se sugiere el protocolo sugerido por Emura (Figura 1)(Evidencia III; Recomendación C).

La EDA dedicada a detectar CG incipiente tiene demandas particulares, que se resumen en la Tabla 6.

La diferencia entre la proporción de CG incipiente diagnosticados en países asiáticos $(50-89 \%)^{8,21,72,73}$ y Chile $(10-20 \%)^{2,10}$ no parece explicable sólo por acceso más fácil a la $\mathrm{EDA}^{74}$, sino también por el uso de una mejor técnica endoscópica. Un estudio japonés mostró que un entrenamiento específico aumenta la tasa de detección de lesiones incipientes ${ }^{75}$.

Tabla 5. Programa de tamizaje endoscópico: Estratificación del riesgo de CG e intervalos de control

\begin{tabular}{|c|c|c|}
\hline Grupo & Riesgo futuro CG & Intervalo de control \\
\hline OLGA/OLGIM 0, H. pylori (-) & Muy bajo & No justifica control (sale del programa) \\
\hline OLGAVOLGIM 0, H. pylori (-), Familiar directo con CG & Bajo & EDA cada 5 años \\
\hline OLGAVOLGIM 0, H. pylori $(+)^{1}$ & Bajo & EDA cada 3 años \\
\hline OLGA/OLGIM I - ॥ & Moderado & EDA cada 3 años \\
\hline OLGA/OLGIM III - IV & Alto & EDA anual \\
\hline $\mathrm{DBG}^{2}$ & Alto & EDA anual \\
\hline $\mathrm{DAG}^{3}$ & Muy alto & EDA cada 6 meses \\
\hline
\end{tabular}

${ }^{1} \mathrm{H}$. pylori refractario a la erradicación + gastritis predominio corporal. ${ }^{2}$ Displasia de bajo grado en biopsias sistemáticas, sin lesión focal detectable. ${ }^{3}$ Displasia de alto grado en biopsias sistemáticas, sin lesión focal detectable. 
La premedicación con anti-burbujas y/o mucolíticos por vía oral antes de la EDA es de uso rutinario en Japón. Varios ensayos clínicos aleatorizados sugieren que pronasa ${ }^{76}$ o dimeticona con $\mathrm{N}$-acetilcisteina (NAC) $)^{77,78}$ disminuyen la necesidad de lavado y acortan el examen. En nuestro medio, es posible utilizar simeticona a $4 \%$ (40-120 mg) y NAC (400 mg) en $80-100 \mathrm{ml}$ de agua, 10-30 min antes de la EDA. Durante el procedimiento se puede utilizar NAC o ácido acético a $1,5 \%$ adicional, pero esto no reemplaza su uso previo $^{79}$

Es importante una distensión gástrica completa, para explorar toda la superficie gástrica. $\mathrm{La}$ utilización de índigo carmín o azul de metileno, la magnificación óptica y el uso de narrow band imaging (NBI) y probablemente otros sistemas de cromoscopia electrónica (FICE o I-Scan) permiten caracterizar mejor las lesiones ${ }^{80,81}$.

En Japón, el registro sistemático de imágenes es parte integral de toda EDA. Una adaptación del sistema japonés, denominado Endoscopia Sistemática Alfanumérica Codificada ${ }^{82}$ (Figura 1) sugiere capturar 28 imágenes predefinidas, que cubren la mayor parte de la superficie mucosa.

\section{Requisitos de un programa de seguimiento endoscópico}

\section{Recomendaciones}

22. Se requiere un equipo de trabajo conformado por endoscopista, patólogo, enfermera y secretaria, capacitados en sus tareas específicas. (Evidencia III, Recomendación C).

23. El reporte definitivo debe incluir doble identificación del paciente, modalidad de contacto, identificación inequívoca de las biopsias, presencia y caracterización de lesiones focales, reporte histológico, conclusión final, categorización del riesgo futuro de CG y fecha del siguiente control (Evidencia III; Recomendación C).

24. Se sugiere un control de calidad basado en los siguientes indicadores:

1. $\mathrm{N}^{\circ}$ de $\mathrm{CG}$ incipientes/ $\mathrm{N}^{\circ}$ total de CG diagnosticados anualmente en la institución.

2. $\mathrm{N}^{\circ}$ de endoscopias con registro fotográfico completo $/ \mathrm{N}^{\circ}$ total de endoscopias de tamizaje.

Se requiere un equipo de trabajo multidisciplinario constituido por endoscopista(s), anatomo-

Tabla 6. Diferencias entre EDA diagnóstica habitual y EDA en programa de tamizaje endoscópico de CG

\begin{tabular}{|c|c|c|}
\hline & EDA Diagnóstica & EDA Tamizaje CG \\
\hline $\begin{array}{l}\text { Objetivos del } \\
\text { procedimiento }\end{array}$ & $\begin{array}{l}\text { Buscar causa de síntomas } \\
\text { Detectar AG / MI }\end{array}$ & $\begin{array}{l}\text { Detectar CG incipiente } \\
\text { Definir riesgo futuro CG }\end{array}$ \\
\hline Preparación & Ayuno & $\begin{array}{l}\text { Ayuno } \\
\text { Mucolíticos pre EDA }\end{array}$ \\
\hline $\begin{array}{l}\text { Equipamiento } \\
\text { necesario }\end{array}$ & Endoscopio estándar & $\begin{array}{l}\text { Endoscopio alta resolución } \\
\text { Disponibilidad tinciones } \\
\text { Deseable cromoscopia digital }\end{array}$ \\
\hline Técnica & $\begin{array}{l}\text { Exploración completa } \\
\text { Test de ureasa } \\
\text { Bx lesiones sospechosas } \\
\text { Bx múltiples (Sydney) (según edad) }\end{array}$ & $\begin{array}{l}\text { Exploración completa } \\
\text { Búsqueda lesiones inaparentes } \\
\text { Cromoscopia (tinción o electrónica) } \\
\text { Bx multiples (Sydney) }\end{array}$ \\
\hline Tiempo requerido & $15-30 \mathrm{~min}$ & $30-45 \mathrm{~min}$ \\
\hline Reporte & $\begin{array}{l}\text { Descripción estándar } \\
\text { Registro imágenes de eventuales lesiones }\end{array}$ & $\begin{array}{l}\text { Descripción estandar } \\
\text { Registro estandarizado de imágenes }\end{array}$ \\
\hline Control & $\begin{array}{l}\text { No programado. } \\
\text { Según patología }\end{array}$ & $\begin{array}{l}\text { Programado } \\
\text { Según riesgo CG estimado }\end{array}$ \\
\hline Endoscopista & Entrenamiento estándar & $\begin{array}{l}\text { Entrenamiento especifico en detección de CG incipiente } \\
\text { Entrenamiento en tinciones y/o cromoscopia digital }\end{array}$ \\
\hline
\end{tabular}




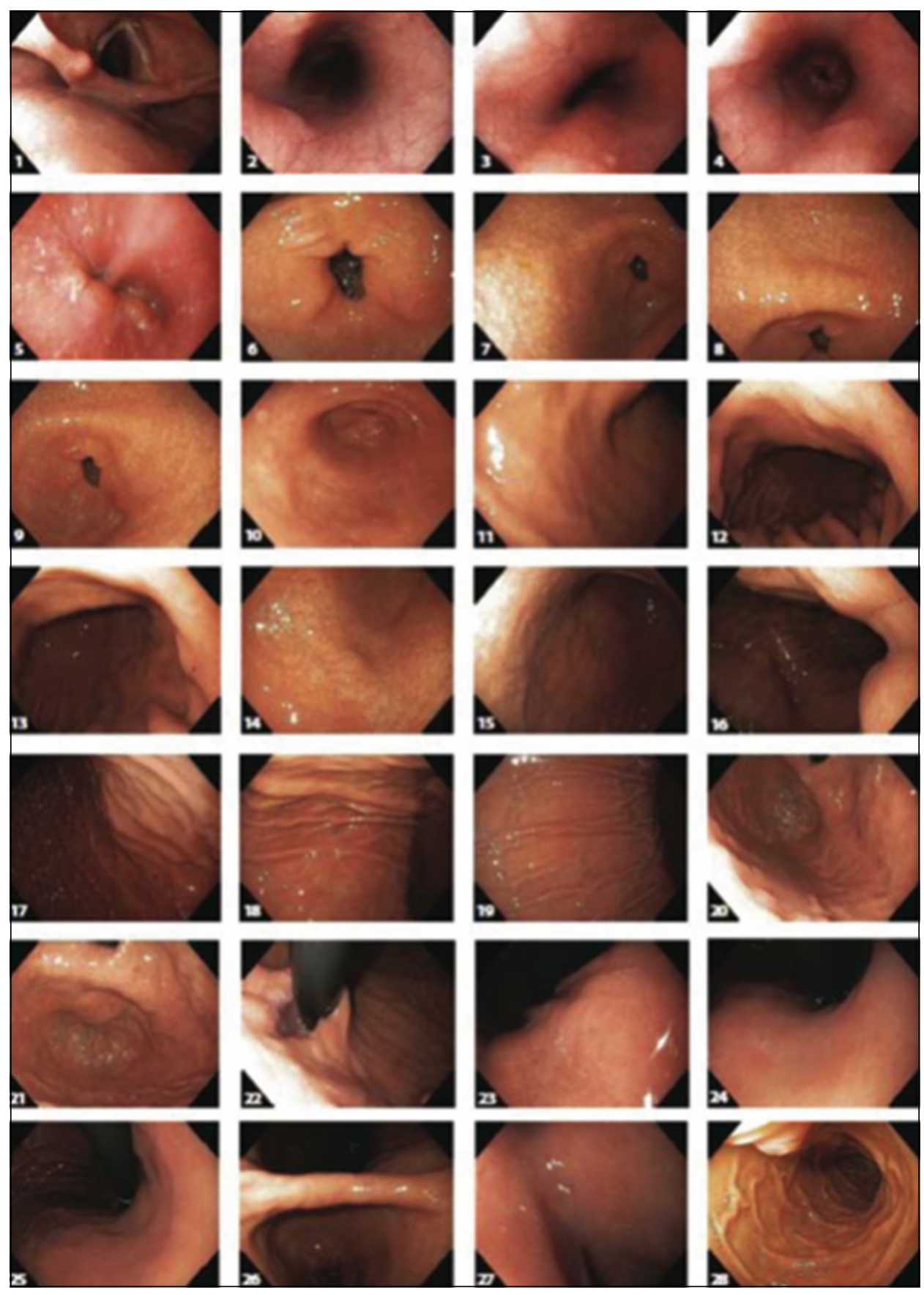

Figura 1. Registro de Imágenes (Endoscopia Sistemática Codificada) (modificado de ref. 82). 1) Hipofaringe; 2) Esófago, tercio superior; 3) Esófago, tercio medio; 4) Esófago, tercio inferior; 5) Hiato esofágico (UGE); 6) Piloro; 7) Antro, pared anterior; 8) Antro, curva menor; 9) Antro, pared posterior; 10) Antro, curva mayor; 11) Cuerpo distal, cara anterior; 12) Cuerpo distal, curva menor; 13) Cuerpo distal, cara posterior; 14) Cuerpo distal, curva mayor; 15) Cuerpo medio, cara anterior; 16) Cuerpo medio, curva menor; 17) Cuerpo medio, cara posterior; 18) Cuerpo medio, curva mayor; 19) Cuerpo alto, curva mayor; 20) Cuerpo alto, pared anterior y posterior; 21) Fondo gástrico; 22) Unión gastroesofágica (retrovisión); 23) Curva menor, tercio superior; 24) Curva menor, tercio medio; 25) Curva menor, tercio distal; 26) Ángulo gástrico; 27) Bulbo duodenal; 28) Duodeno, segunda porción; 
Protocolo ACHED para manejo de lesiones premalignas gástricas - A. Rollán et al

patólogo, enfermera y secretaria formalmente capacitados.

El informe histológico está disponible semanas después de la EDA. Debe implementarse un proceso administrativo para rescatar el informe (secretaria), definir la conducta e intervalo de control (endoscopista), registrarlo en el reporte definitivo (endoscopista), agendar al paciente (enfermera) y confirmar su fecha de citación (secretaria).

Respecto a cáncer colorectal se ha demostrado una significativa variación interindividual en la tasa de detección de pólipos colónicos y se han consensuado criterios de calidad ${ }^{84}$. Respecto a CG probablemente exista una variabilidad similar. El objetivo central es aumentar la detección de CG incipiente. La relación entre el número de CG incipientes y CG totales diagnosticados (Yao K, comunicación personal) es simple y fácil de calcular y permite definir metas progresivas, ajustadas a la realizada local. El registro sistemático de imagenes asegura el examen completo y pudiera aumentar la probabilidad de detectar lesiones. Probablemente se requiere un entrenamiento específico.

\section{Conclusiones}

Nuestra propuesta complementa el protocolo AUGE para mejorar el diagnóstico precoz de CG. Propone biopsias gástricas sistemáticas y calificación individual del riesgo de CG. Esto puede implementarse con los recursos actualmente disponibles. Deben realizarse estudios de costo-efectividad, pero el aumento de biopsias puede ser más que compensado evitando nuevas endoscopias en el grupo de bajo riesgo. Estudiando 100 pacientes consecutivos del sector oriente de Santiago, sólo $8 \%$ de ellos era portador de AG intensa, tributarios de seguimiento endoscópico anual (Rollán A. Datos no publicados).

Un programa de seguimiento endoscópico exige la destinación y capacitación de recursos humanos y materiales, pero es factible en cualquier servicio de endoscopia digestiva. El protocolo involucra a diferentes actores. Los endoscopistas deben tomar biopsias sistemáticas y realizar una exploración dedicada a detectar lesiones incipientes. Los patólogos deben utilizar protocolo de Sydney e incluir en el reporte la etapificación OLGA/ OLGIM. Los servicios de endoscopia y anatomía patológica deben generar procesos administrati- vos que aseguren la notificación al paciente y su eventual citación al control endoscópico. Podría complementarse con programas dirigidos a población asintomática, lo que es actualmente es factible por métodos serológicos.

Aun cuando la evidencia y el consenso sugieren que estas recomendaciones pueden mejorar el pronóstico del CG en Chile, la mayoría tienen grados de evidencia y recomendación inciertas, revelando la necesidad de generar información de buena calidad. La aplicación de este protocolo puede contribuir a este propósito.

\section{Referencias}

1. Bertuccio P, Chatenoud L, Levi F, Praud D, Ferlay J, Negri E, et al. Recent patterns in gastric cancer: a global overview. Int J Cancer 2009; 125 (3): 666-73.

2. Cenitagoya GF, Bergh CK, Klinger-Roitman J. A prospective study of gastric cancer. "Real" 5-year survival rates and mortality rates in a country with high incidence. Dig Surg 1998; 15 (4): 317-22.

3. Heise K, Bertran E, Andia ME, Ferreccio C. Incidence and survival of stomach cancer in a high-risk population of Chile. World J Gastroenterol 2009; 15 (15): 1854-62.

4. Correa P. Gastric cancer: overview. Gastroenterol Clin North Am 2013; 42 (2): 211-7.

5. Correa P, Cuello C, Duque E. Carcinoma and intestinal metaplasia of the stomach in Colombian migrants. J Natl Cancer Inst 1970; 44 (2): 297-306.

6. Hamashima C, Shibuya D, Yamazaki H, Inoue K, Fukao A, Saito H, et al. The Japanese guidelines for gastric cancer screening. Jpn J Clin Oncol 2008; 38 (4): 259-67.

7. Leung WK, Wu M, Kakugawa Y, Kim JJ, Yeoh K, Goh $\mathrm{KL}$, et al. Screening for gastric cancer in Asia: current evidence and practice. Lancet Oncol 2008; 9 (3): 279-87.

8. Yuan Y. A survey and evaluation of population-based screening for gastric cancer. Cancer Biol Med 2013; 10 (2): 72-80.

9. Llorens P. Gastric cancer mass survey in Chile. Semin Surg Oncol 1991; 7 (6): 339-43.

10. Calvo Belmar A, Pruyas M, Nilsen E, Verdugo P. [Populational research of gastric cancer in digestive symptomatic patients, from 1996 to 2000]. Rev Med Chile 2001; 129 (7): 749-55.

11. Harris RP, Helfand M, Woolf SH, Lohr KN, Mulrow $\mathrm{CD}$, Teutsch SM, et al. Current methods of the US Preventive Services Task Force: a review of the process. Am J Prev Med 2001; 20 (3 Suppl): 21-35.

12. Sawaya GF, Guirguis-Blake J, LeFevre M, Harris R, Petitti D. Update on the methods of the U.S. Preventive 
Services Task Force: estimating certainty and magnitude of net benefit. Ann Intern Med 2007; 147 (12): 871-5.

13. Hasson F, Keeney S, McKenna H. Research guidelines for the Delphi survey technique. J Adv Nurs 2000; 32 (4): 1008-15.

14. Graham D Y, Asaka M. Eradication of gastric cancer and more efficient gastric cancer surveillance in Japan: two peas in a pod. J Gastroenterol 2010; 45 (1): 1-8.

15. Uemura N, Okamoto S, Yamamoto S, Matsumura N, Yamaguchi S, Yamakido M, et al. Helicobacter pylori infection and the development of gastric cancer. $\mathrm{N} \mathrm{Engl}$ J Med 2001; 345 (11): 784-9.

16. Ohata H, Kitauchi S, Yoshimura N, Mugitani K, Iwane M, Nakamura H, et al. Progression of chronic atrophic gastritis associated with Helicobacter pylori infection increases risk of gastric cancer. Int J Cancer 2004; 109 (1): 138-43.

17. Fuccio L, Eusebi LH, Bazzoli F. Gastric cancer, Helicobacter pylori infection and other risk factors. World J Gastrointest Oncol 2010; 2 (9): 342-7.

18. Fukase K, Kato M, Kikuchi S, Inoue K, Uemura N, Okamoto S, et al. Effect of eradication of Helicobacter pylori on incidence of metachronous gastric carcinoma after endoscopic resection of early gastric cancer: an open-label, randomised controlled trial. Lancet 2008; 372 (9636): 392-7.

19. Ferreccio C, Rollán A, Harris PR, Serrano C, Gederlini A, Margozzini P, et al. Gastric cancer is related to early Helicobacter pylori infection in a high-prevalence country. Cancer Epidemiol. Biomarkers Prev 2007; 16 (4): 662-7.

20. Ortega JP, Espino A, Calvo B A, Verdugo P, Pruyas M, Nilsen E, et al. Helicobacter pylori infection in symptomatic patients with benign gastroduodenal diseases: analysis of 5.664 cases. Rev Med Chile 2010; 138: 529-35.

21. Fock KM, Katelaris P, Sugano K, Ang TL, Hunt R, Talley NJ, et al. Second Asia-Pacific Consensus Guidelines for Helicobacter pylori infection. J Gastroenterol Hepatol 2009; 24 (10): 1587-600.

22. Malfertheiner P, Megraud F, O’Morain CA, Atherton J, Axon ATR, Bazzoli F, et al. Management of Helicobacter pylori infection-the Maastricht IV/ Florence Consensus Report. Gut 2012; 61 (5): 646-64.

23. Rollan A, Ferreccio C, Gederlini A, Serrano C, Torres J, Harris P. Non-invasive diagnosis of gastric mucosal atrophy in an asymptomatic population with high prevalence of gastric cancer. World J Gastroenterol 2006; 12 (44): 7172-8.

24. Dinis-Ribeiro M, Areia M, Vries AC De, Pereira C, Correia R, Ensari A, et al. Management of precancerous conditions and lesions in the stomach (MAPS ). Guide- line from the European Society of Gastrointestinal Endoscopy (ESGE), European Helicobacter Study Group (EHSG), European Society of Pathology (ESP), and the Sociedade Portuguesa de Endoscopia Digestiva (SPED). Endoscopy 2012; 44 (1): 74-94.

25. La Vecchia C, Negri E, Franceschi S, Gentile A. Family history and the risk of stomach and colorectal cancer. Cancer 1992; 70 (1): 50-5.

26. Yaghoobi M, Bijarchi R, Narod SA. Family history and the risk of gastric cancer. Br J Cancer 2010; 102 (2): 23742.

27. El-Omar EM, Oien K, Murray LS, El-Nujumi A, Wirz A, Gillen D, et al. Increased prevalence of precancerous changes in relatives of gastric cancer patients: critical role of $H$. pylori. Gastroenterology 2000; 118 (1): 22-30.

28. Marcos-Pinto R, Carneiro F, Dinis-Ribeiro M, Wen X, Lopes C, Figueiredo C, et al. First-degree relatives of patients with early-onset gastric carcinoma show even at young ages a high prevalence of advanced OLGA/ OLGIM stages and dysplasia. Aliment Pharmacol Ther 2012; 35 (12): 1451-9.

29. Rugge M. Secondary prevention of gastric cancer. Gut 2007; 56 (12): 1646-7.

30. Kashiwagi H. Ulcers and gastritis. Endoscopy 2005; 37 (2): 110-5.

31. Lim JH, Kim N, Lee HS, Choe G, Jo SY, Chon I, et al. Correlation between Endoscopic and Histological Diagnoses of Gastric Intestinal Metaplasia. Gut Liver 2013; 7 (1): 41-50.

32. Graham DY, Kato M, Asaka M. Gastric endoscopy in the 21st century: appropriate use of an invasive procedure in the era of non-invasive testing. Dig. Liver Dis 2008; 40 (7): 497-503.

33. Dixon MF, Genta RM, Yardley JH, Correa P. Classification and grading of gastritis. The updated Sydney System. International Workshop on the Histopathology of Gastritis, Houston 1994. Am J Surg Pathol 1996; 20 (10): 1161-81.

34. De Vries AC, Haringsma J, de Vries RA, Ter Borg F, van Grieken NCT, Meijer GA, et al. Biopsy strategies for endoscopic surveillance of pre-malignant gastric lesions. Helicobacter 2010; 15 (4): 259-64.

35. Ezoe Y, Muto M, Uedo N, Doyama H, Yao K, Oda I, et al. Magnifying narrowband imaging is more accurate than conventional white-light imaging in diagnosis of gastric mucosal cancer. Gastroenterology 2011; 141 (6): 2017-25.

36. Ekström AM, Held M, Hansson L-E, Engstrand L, Nyrén O. Helicobacter pylori in Gastric Cancer Established by CagA Immunoblot as a Marker of Past Infection. Gastroenterology 2001; 121 (4): 784-91. 
37. Matsuhisa T, Matsukura N, Yamada N. Topography of chronic active gastritis in Helicobacter pylori-positive Asian populations: age-, gender-, and endoscopic diagnosis-matched study. J. Gastroenterol 2004;39(4):324-8.

38. Mukoubayashi C, Yanaoka K, Ohata H, Arii K, Tamai $\mathrm{H}$, Oka M, et al. Serum Pepsinogen and Gastric Cancer Screening. Intern. Med 2007; 46 (6): 261-6.

39. De Vries AC, van Grieken NCT, Looman CWN, Casparie MK, de Vries E, Meijer GA, et al. Gastric cancer risk in patients with premalignant gastric lesions: a nationwide cohort study in the Netherlands. Gastroenterology 2008; 134 (4): 945-52.

40. Coma del Corral MJ, Pardo-Mindan FJ, Razquin S, Ojeda C. Risk of cancer in patients with gastric dysplasia. Follow-up study of 67 patients. Cancer 1990; 65 (9): 2078-85.

41. Sarela AI, Scott N, Verbeke CS, Wyatt JI, Dexter SPL, Sue-Ling HM, et al. Diagnostic variation and outcome for high-grade gastric epithelial dysplasia. Arch Surg 2005; 140 (7): 644-9.

42. Schlemper RJ, Itabashi M, Kato Y, Lewin KJ, Riddell $\mathrm{RH}$, Shimoda T, et al. Differences in diagnostic criteria for gastric carcinoma between Japanese and western pathologists. Lancet 2007; 349 (9067): 1725-9.

43. Schlemper RJ, Riddell RH, Kato Y, Borchard F, Cooper HS, Dawsey SM, et al. The Vienna classification of gastrointestinal epithelial neoplasia. Gut 2000; 47 (2): 251-5.

44. Lauwers GY, Carneiro F, Graham DY et al. Gastric carcinoma. In: Bosman FT, Carneiro F, Hruban RH, Theise ND (eds.) WHO Classification of tumours of the digestive system. 4 edn. Lyon: IARC Press; 2010; 48-58.

45. Meining A, Bayerdörffer E, Müller P, Miehlke S, Lehn N, Hölzel D, et al. Gastric carcinoma risk index in patients infected with Helicobacter pylori. Virchows Arch 1998; 432 (4): 311-4.

46. Rugge M, Genta RM. Staging gastritis: an international proposal. Gastroenterology 2005; 129 (5): 1807-8.

47. el-Zimaity HM, Graham DY, al-Assi MT, Malaty H, Karttunen TJ, Graham DP, et al. Interobserver variation in the histopathological assessment of Helicobacter pylori gastritis. Hum Pathol 1996; 27 (1): 35-41.

48. Capelle LG, de Vries AC, Haringsma J, Ter Borg F, de Vries RA, Bruno MJ, et al. The staging of gastritis with the OLGA system by using intestinal metaplasia as an accurate alternative for atrophic gastritis. Gastrointest. Endosc 2010; 71 (7): 1150-8.

49. Rugge M, Fassan M, Pizzi M, Farinati F, Sturniolo GC, Plebani M, et al. Operative link for gastritis assessment vs operative link on intestinal metaplasia assessment. World J. Gastroenterol 2011; 17 (41): 4596-601.
50. Rollan A. OLGA System ("Operative Link for Gastritis Assessment”). Gastroenterol latinoam 2012; 23 (2): 53 4 .

51. Rugge M, Boni MDE, Lli GP, Bona MDE, Giacomelli L, Fassan M. Gastritis OLGA-staging and gastric cancer risk: a twelve-year clinico-pathological follow-up study. Aliment Pharmacol Ther 2010; 31 (10): 1104-11.

52. Rugge M, Kim JG, Mahachai V, Miehlke S, Pennelli $\mathrm{G}$, Russo VM, et al. OLGA gastritis staging in young adults and country-specific gastric cancer risk. Int J Surg Pathol 2008; 16 (2): 150-4.

53. Satoh K, Osawa H, Yoshizawa M, Nakano H, Hirasawa $\mathrm{T}$, Kihira K, et al. Assessment of atrophic gastritis using the OLGA system. Helicobacter 2008; 13 (3): 225-9.

54. Carrasco G, Corvalan AH. Helicobacter pylori-Induced Chronic Gastritis and Assessing Risks for Gastric Cancer. Gastroenterol Res Pract 2013; 2013: 393015.

55. Tsai Y-C, Hsiao W-H, Yang H-B, Cheng H-C, Chang $\mathrm{W}-\mathrm{L}, \mathrm{Lu} \mathrm{C}-\mathrm{C}$, et al. The corpus-predominant gastritis index may serve as an early marker of Helicobacter pylori-infected patients at risk of gastric cancer. Aliment Pharmacol Ther 2013; 37 (10): 969-78.

56. Rollan A, Giancaspero R, Fuster F, Acevedo C, Figueroa $\mathrm{C}$, Hola $\mathrm{K}$, et al. The long-term reinfection rate and the course of duodenal ulcer disease after eradication of Helicobacter pylori in a developing country. Am J Gastroenterol 2000; 95 (1): 50-6.

57. Malfertheiner P. Gastric Atrophy Reversible or Irreversible after Helicobacter pylori Eradication - An Open Question. Digestion 2011; 83 (4): 250-2 2011; 250-2.

58. Wang J, Xu L, Shi R, Huang X, Li SWH, Huang Z, et al. Gastric atrophy and intestinal metaplasia before and after Helicobacter pylori eradication: a meta-analysis. Digestion 2011; 83 (4): 253-60.

59. You W, Brown LM, Zhang L, Li J, Jin M, Chang Y, et al. Randomized double-blind factorial trial of three treatments to reduce the prevalence of precancerous gastric lesions. J. Natl. Cancer Inst 2006; 98 (14): 974-83.

60. Fuccio L, Zagari RM, Eusebi LH, Laterza L, Cennamo V, Ceroni L, et al. Meta-analysis: can Helicobacter pylori eradication treatment reduce the risk for gastric cancer? Ann. Intern. Med 2009;151(2):121-8.

61. Take S, Mizuno M, Ishiki K, Yoshida T, Ohara N, Yokota $\mathrm{K}$, et al. The long-term risk of gastric cancer after the successful eradication of Helicobacter pylori. J Gastroenterol 2011; 46 (3): 318-24.

62. Whiting JL, Sigurdsson A, Rowlands DC, Hallissey MT, Fielding JWL. The long term results of endoscopic surveillance of premalignant gastric lesions. Gut 2002; 50 (3): 378-81.

63. Dan YY, So JBY, Yeoh KG. Endoscopic screening for 
gastric cancer. Clin. Gastroenterol. Hepatol 2006; 4 (6): 709-16.

64. Shin WG, Kim HU, Song HJ, Hong SJ, Shim K-N, Sung $\mathrm{I}-\mathrm{K}$, et al. Surveillance strategy of atrophic gastritis and intestinal metaplasia in a country with a high prevalence of gastric cancer. Dig Dis Sci 2012; 57 (3): 746-52.

65. Zullo A, Hassan C, Romiti A, Giusto M, Guerriero C, Lorenzetti R, et al. Follow-up of intestinal metaplasia in the stomach: When, how and why. World J. Gastrointest. Oncol 2012; 4 (3): 30-6.

66. Yeh JM, Hur C, Kuntz KM, Ezzati M, Goldie SJ. Costeffectiveness of treatment and endoscopic surveillance of precancerous lesions to prevent gastric cancer. Cancer 2010; 116 (12): 2941-53.

67. Hassan C, Zullo A, Di Giulio E, Annibale B, Lahner E, De Francesco V, et al. Cost-effectiveness of endoscopic surveillance for gastric intestinal metaplasia. Helicobacter 2010; 15 (3): 221-6.

68. Dixon MF. Gastrointestinal epithelial neoplasia: Vienna revisited. Gut 2002; 51 (1): 130-1.

69. Kotwal AA, Mohile SG, Dale W. Remaining Life Expectancy Measurement and PSA Screening of Older Men. J Geriatr Oncol 2012; 3 (3): 196-204.

70. Qaseem A, Denberg TD, Jr RHH, Humphrey LL, Levine J. Screening for Colorectal Cancer: A Guidance Statement From the. Ann Intern Med 2012; 156 (5): 378-86.

71. Qaseem A, Barry MJ, Denberg TD, Owens DK, Shekelle P. Screening for prostate cancer: a guidance statement from the Clinical Guidelines Committee of the American College of Physicians. Ann Intern Med 2013; 158 (10): 761-9.

72. Matsumoto S, Yamasaki K, Tsuji K, Shirahama S. Results of mass endoscopic examination for gastric cancer in Kamigoto Hospital, Nagasaki Prefecture. World J Gastroenterol 2007; 13 (32): 4316-20.

73. Jung K-W, Park S, Kong H-J, Won Y-J, Boo Y-K, Shin $\mathrm{H}-\mathrm{R}$, et al. Cancer statistics in Korea: incidence, mortality and survival in 2006-2007. J Korean Med Sci 2010; 25 (8): 1113-21.

74. Suvakovic Z, Bramble MG, Jones R, Wilson C, Idle N, Ryott J. Improving the detection rate of early gastric cancer requires more than open access gastroscopy: a five year study. Gut 1997; 41 (3): 308-13.
75. Yamazato T, Oyama T, Yoshida T, Baba Y, Yamanouchi $\mathrm{K}$, Ishii Y, et al. Two Years' Intensive Training in Endoscopic Diagnosis Facilitates Detection of Early Gastric Cancer Intern Med 2012; 51 (12): 1461-5.

76. Lee GJ, Park SJ, Kim SJ, Kim HH, Park MI, Moon W. Effectiveness of Premedication with Pronase for Visualization of the Mucosa during Endoscopy: A Randomized, Controlled Trial. Clin Endosc 2012; 45 (2): 161-4.

77. Neale JR, James S, Callaghan J, Patel P. Premedication with $\mathrm{N}$-acetylcysteine and simethicone improves mucosal visualization during gastroscopy: a randomized, controlled, endoscopist-blinded study. Eur J Gastroenterol Hepatol 2013; 25 (7): 778-83.

78. Chen M-J, Wang H-Y, Chang C-W, Hu K-C, Hung C-Y, Chen C-J, et al. The add-on $\mathrm{N}$-acetylcysteine is more effective than dimethicone alone to eliminate mucus during narrow-band imaging endoscopy: a double-blind, randomized controlled trial. Scand. J. Gastroenterol 2013; 48 (2): 241-5.

79. Bhandari P, Green S, Hamanaka H, Nakajima T, Matsuda T, Saito Y, et al. Use of Gascon and Pronase either as a pre-endoscopic drink or as targeted endoscopic flushes to improve visibility during gastroscopy: a prospective, randomized, controlled, blinded trial. Scand. J. Gastroenterol 2010; 45 (3): 357-61.

80. Kato M, Kaise M, Yonezawa J, Toyoizumi H, Yoshimura N, Yoshida Y, et al. Magnifying endoscopy with narrowband imaging achieves superior accuracy in the differential diagnosis of superficial gastric lesions identified with white-light endoscopy: a prospective study. Gastrointest Endosc 2010; 72 (3): 523-9.

81. Zhang J, Guo S-B, Duan Z-J. Application of magnifying narrow-band imaging endoscopy for diagnosis of early gastric cancer and precancerous lesion. BMC Gastroenterol 2011; 11: 135.

82. Emura F, Gralnek I, Baron TH. Improving early detection of gastric cancer: a novel systematic alphanumericcoded endoscopic approach. Rev. Gastroenterol. Peru 2013; 33 (1): 52-8.

83. Lee TJW, Rutter MD, Blanks RG, Moss SM, Goddard AF, Chilton A, et al. Colonoscopy quality measures: experience from the NHS Bowel Cancer Screening Programme. Gut 2012; 61 (7): 1050-7. 